\section{Surgical outcomes for vitrectomy in Eales' disease}

D Shukla', S Kanungo', NM Prasad² and R Kim ${ }^{1}$

Keywords: vitrectomy; Eales'disease; vitreous haemorrhage

Aim To present and analyse the anatomical and functional outcomes for vitrectomy in Eales' disease.

Methods This retrospective case series enrolled 63 patients (71 eyes) who underwent pars plana vitrectomy (PPV) for the complications of Eales' disease. Indications included nonclearing vitreous haemorrhage (VH) with/without epiretinal membranes in $49(69 \%)$ eyes, and retinal detachments (RD) involving/threatening macula in $22(31 \%)$. Additional procedures (scleral buckling; gas/oil tamponade) were performed at surgeon's discretion. Minimum follow-up was 6 months. The primary outcome measures were change in best-corrected visual acuity (BCVA) and posterior segment status. Results The mean age of the patients was 29.6 years (range: 15-70 years); 60 were male (95\%). Preoperatively, posterior vitreous detachment (PVD) existed in 28 (39.4\%) eyes. Forty (56.3\%)

${ }^{1}$ Retina-Vitreous Service, Aravind Eye Hospital and Postgraduate Institute of Ophthalmology, Madurai, Tamil Nadu, India

${ }^{2}$ Department of Biostatistics, Lions Aravind Institute of Community Ophthalmology, Madurai, Tamil Nadu, India

Correspondence: D Shukla, Aravind Eye Hospital and Postgraduate Institute of Ophthalmology, 1 Anna Nagar, Madurai 625 020, Tamil Nadu, India

Tel: + 914524356100 ;

Fax: + 914522530984

E-mails: daksh@

aravind.org or daksh66@ gmail.com

Received: 28 August 2006 Accepted in revised form: 31 December 2006 Published online: 30 March 2007 eyes underwent only PPV; the rest required additional surgical procedures (q.v.). The mean baseline BCVA (1/60) improved to 6/24 postoperatively $(P<0.0001)$. Fifty-four eyes of 50 patients $(76 \%)$ showed an improvement of $\geqslant 2$ equivalent Snellen lines; six eyes (four patients) remained stable ( \pm 1 line); visual acuity worsened in 11 eyes (nine patients). The mean final BCVA was similar in eyes operated for VH and RD $(P=0.08)$; but the magnitude of change from baseline was greater in the VH group $(P=0.009)$. PVD had a borderline association with final BCVA $(P=0.056)$; but did not influence the functional/anatomical improvement. Thirteen eyes required repeat interventions; 11 (15.49\%) eyes experienced surgical failure.

Conclusions Although surgical outcomes in Eales' disease depend on preoperative PVD/RD to some extent; good results are possible in the presence of incomplete PVD and tractional sequelae. Eye (2008) 22, 900-904; doi:10.1038/sj.eye.6702763; published online 30 March 2007
Introduction

Eales' disease is an idiopathic, occlusive periphlebitis affecting peripheral retina in young men, leading to retinal nonperfusion and neovascularisation, with subsequent vitreous haemorrhage $(\mathrm{VH})$, and tractional complications. ${ }^{1-3}$ The disease is observed more commonly in the Indian subcontinent than in the rest of the world. ${ }^{1,2,4}$ The management depends on the stage of the disease: medical treatment with oral corticosteroids in the active inflammatory stage, and laser photocoagulation in presence of retinal ischaemia and neovascularisation. Some cases require vitreoretinal surgery for $\mathrm{VH}$ and tractional complications; surgical results have been variable in the published literature. ${ }^{1,5-8}$ Though satisfactory overall results have been claimed, ${ }^{1}$ the reported outcomes differ considerably with the surgical indication $(\mathrm{VH}$ vs tractional complications), the extent of posterior vitreous detachment (PVD), and even surgical techniques. ${ }^{5-10}$ Some of the largest surgical studies are over two decades' old; $;, 8$ the recent ones are small. 5,6,9-11 The effect of surgical variables like PVD, retinal detachments (RD), and intraoperative procedures on the final outcome have not been evaluated in a controlled manner. We present our long-term surgical experience with Eales' disease; and analyse the influence of preoperative and surgical variables on anatomical and visual outcome.

Materials and methods

Patient profile

Clinical data on the surgical in-patients admitted with a diagnosis of primary retinal 
vasculitis at a tertiary eye care centre over an 8-year period (January 1998-2006) were retrieved from their case records. The clinical diagnosis of Eales' disease was made on the basis of peripheral venous sheathing in multiple quadrants in the fellow eye or the operated eye (observed intraoperatively). The indications for vitrectomy included best-corrected visual acuity (BCVA) less than 6/12 owing to $\mathrm{VH}$ of at least 2 months' duration with/without epiretinal membranes; rhegmatogenous / combined-mechanism RD; or a tractional retinal detachment involving/threatening the macula. Patients with associated conditions such as noninflammatory retinal vein occlusions, diabetic or hypertensive retinopathy, sickle cell retinopathy; ocular inflammatory conditions like choroiditis, pars planitis, disc oedema etc; or associated systemic infective/autoimmune/other inflammatory disease revealed by history, examination, or investigations were excluded form the analysis, as were patients with a follow-up of less than 6 months after the last surgical procedure.

\section{Patient work-up}

Demographic, medical, and ophthalmic data were recorded in detail. Demographic information included age, sex, race, place of residence, occupation, travel/ treatment history. Ophthalmic evaluation included determination of BCVA, applanation tonometrey, slitlamp biomicroscopy, and indirect ophthalmoscopy. B-scan ultrasonography was performed on all patients to determine the status of the retina and extent of PVD. Investigations performed for all cases included a complete haemogram, fasting blood sugar, peripheral smear, erythrocyte sedimentation rate, Mantoux test, chest X-ray, treponemal antibody test, enzyme-linked immunosorbent assay for human immunodeficiency virus, and urine analysis. Specific investigations like antinuclear antibody testing were reserved for cases where history or clinical examination raised the level of suspicion towards a specific aetiological diagnosis. Informed consent was obtained from each patient after explaining the nature of the disease and surgical details. The study was approved by the Institutional Review Board of the Aravind Eye Care System.

\section{Treatment, follow-up, and outcome measures}

All patients underwent pars plana vitrectomy (PPV) and endophotocoagulation, with/without additional procedures like scleral belt buckling using a No. 42 or No. 240 silicone encircling band (SB), perfluoropropane gas $\left(\mathrm{C}_{3} \mathrm{~F}_{8}\right)$, or silicone oil tamponade, at the surgeon's discretion. Patients were advised follow-up at intervals of 1,3 , and 6 months, and yearly thereafter. Silicone oil
Table 1 Visual acuity score preoperatively, at 6 months and at final follow-up

\begin{tabular}{llccc}
\hline \multirow{2}{*}{$\begin{array}{l}\text { Snellen } \\
\text { acuity }\end{array}$} & \multirow{2}{*}{$\begin{array}{l}\text { Visual } \\
\text { acuity score }\end{array}$} & \multicolumn{3}{c}{ Number of eyes (\%) } \\
\cline { 3 - 5 } & & Baseline & 6 months & $\begin{array}{c}\text { Final } \\
\text { follow-up }\end{array}$ \\
\hline $6 / 6-6 / 12$ & $1-3$ & $3(4.23)$ & $32(50.00)$ & $43(60.56)$ \\
$6 / 18-6 / 36$ & $4-6$ & $5(7.04)$ & $9(14.06)$ & $10(14.08)$ \\
$6 / 60-4 / 60$ & $7-9$ & $8(11.27)$ & $7(10.94)$ & $2(2.82)$ \\
$3 / 60-1 / 60$ & $10-12$ & $17(23.94)$ & $5(7.81)$ & $4(5.63)$ \\
$1 / 120$, FCF, & $13,14,15$ & $35(49.29)$ & $5(7.81)$ & $2(2.82)$ \\
HM & & & & \\
PL, no PL & 16,17 & $3(4.23)$ & $6(9.38)$ & $10(14.08)$ \\
Total & $1-17$ & $71(100.00)$ & $64(100.00)$ & $71(100.00)$ \\
\end{tabular}

Abbreviations: FCF, finger counting close to face; HM, hand motions; PL, perception of light.

was removed 3-6 months postoperatively. The primary outcome measures were change in BCVA and retinal status.

\section{Statistical analysis}

Visual acuity as measured using Snellen chart ranged from $6 / 6$ to light perception at presentation. For ease of analysis, equivalent scores were assigned to each level of visual acuity, as depicted in Table 1.

Statistical analysis was performed using STATA version 7.0 software package (College Station, TX, USA). Two-sample $t$-test was used to analyse postoperative change in BCVA; and for intergroup comparisons of visual outcome between patients with $\mathrm{VH}$ vs those with $\mathrm{RD}$, between cases requiring vitrectomy alone $v$ s those needing additional procedures, and between cases with partial PVD vs total PVD.

\section{Results}

The study included 71 eyes of 63 patients. Sixty (95\%) patients were male. Age ranged from 15 to 70 years (mean: 29.6 years). Mantoux positivity was observed in all the patients. The indications for vitrectomy were nonclearing VH (49 (69\%) eyes), and secondary RD (macular and extramacular; rhegmatogenous and combined-mechanism detachments) (22 (31\%) eyes). None of the patients had active vasculitis in either eye. Ultrasonography showed a total PVD in 28 (39.4\%) eyes; and partial or no PVD in the rest. Preoperative panretinal photocoagulation (PRP) marks were present in 21(29.6\%) eyes. PPV with scatter endophotocoagulation was performed in $40(56.3 \%)$ eyes; the rest required additional surgical procedures $\left(\mathrm{SB} / \mathrm{C}_{3} \mathrm{~F}_{8} /\right.$ silicone oil).

The mean baseline BCVA score $(12.11 ; 95 \%$ CI: 11.22 , 3.00 ) corresponded to a Snellen acuity of $1 / 60$. At 
6-month postoperative visit, data were available on 58 patients (64 eyes); five patients followed up after variable time intervals (8-14 months). At the final follow-up, mean BCVA had significantly improved to 5.24 (95\% CI: $3.89,6.59)$, that is, approximately, $6 / 24$ $(P<0.0001)$. Fifty-four eyes of 50 patients $(76 \%)$ showed an improvement in visual acuity, six eyes of four patients remained stable (change equivalent to 1 line); and the visual acuity worsened in 11 eyes of nine patients.

The mean final BCVA of eyes operated for $\mathrm{VH}$ was only marginally better than that of eyes operated for RD (scores equivalent to Snellen acuity of 6/18p and 6/60, respectively, $P=0.08)$. However, the $\mathrm{VH}$ group scored significantly better than the RD group (8.3 vs 3.7 lines, $P=0.009$ ) in terms of mean visual improvement.

Unpaired $t$-test comparing the difference in the mean final visual acuity scores of the group that underwent a relatively simple surgical procedure ( $n=44$; PPV with or without the use of gas tamponade; mean score 3.273) and the group that underwent more complicated procedures ( $n=27$; PPV with scleral buckling and/or silicone oil tamponade; mean score 8.445$)$ showed a significant difference ( $P=0.0001)$ between the two groups. There was also a significant difference in the mean change of visual acuity scores from baseline between the two groups (mean scores 9.296 and 2.926; $P=0.0001$ ). Linear regression was performed to determine the extent to which the final visual acuity score depended on baseline visual acuity (adjusted $r^{2}=-0.01, P=0.74$ ), and on pre-existing retinal photocoagulation (adjusted $\left.r^{2}=-0.01, P=0.68\right)$. No significant association was present between either of these variables and the visual outcomes. Presence of total PVD in the operated eyes resulted in a better mean final BCVA in comparison to eyes with partial or no PVD (scores equivalent to Snellen visual acuities of $6 / 12 \mathrm{p}$ vs $6 / 36$, respectively; $P=0.056$ ). However, the requirement of additional procedures (SB, oil/gas tamponade), final retinal status, and visual improvement were not dependent on the extent of PVD.

Thirteen eyes required a second surgical intervention: vitrectomy/vitreous lavage, barrage photocoagulation, $\mathrm{SB}$ and gas/oil tamponade, and anterior retinal cryopexy. The indications for resurgery included recurrent VH (5), new/recurrent RD (7), or neovascular glaucoma (1). Follow-up ranged from 6 to 96 months, averaging 32.8 months (95\% CI: 27.22, 38.35). Seventeen eyes (24\%) developed cataract over the follow-up, and underwent cataract extraction with intraocular lens implantation. In spite of repeated surgical procedures, three patients (three eyes) developed inoperable redetachments. Overall, nine patients (11 eyes (15.5\%)) were surgical failures: six developed inoperable RD, two were phthisical without $\mathrm{RD}$, and three refused further intervention for operable RD. Of these 11 surgical failures, five had been initially operated for $\mathrm{VH}$, and six for RD. Five eyes had undergone PPV with endophotocoagulation as the primary procedure, without any buckling or tamponade.

\section{Discussion}

We performed vitrectomy with or without adjuvant procedures on 71 eyes of 63 patients with complications of Eales' disease. Anatomical success (clear media, attached retina) was obtained in $84.5 \%$ cases, and functional improvement was achieved in $76 \%$. The issue of reporting visual improvement, that is, quantifying visual acuity, is generally addressed by using decimal or $\log$ MAR equivalents for statistical analysis. However, when the majority of the sample falls into categories in the severely visually impaired to blind range ('Count Fingers' or less), no quantitative measures are available to gauge and compare visual acuities. We therefore adopted a system of scoring wherein the means were reported as scores, and indicated the equivalent visual acuity in these situations, as has been reported previously. $^{12}$

The indication for surgery, status of PVD, and complexity of the surgical procedure performed affected the visual outcome in this series; baseline visual acuity and preoperative PRP did not. It is possible that 'complex procedures' were performed on eyes with $\mathrm{RD} /$ incomplete PVD and that these were factors that determined the visual outcome rather than the procedure itself. Thirteen eyes required a repeat intervention, of which three were anatomical failures. It is noteworthy that among the failed interventions (11), nearly half were operated for only $\mathrm{VH}$; and a similar number were performed without complex adjunct procedures.

Results of vitrectomy in Eales' disease have been reported for about three decades. ${ }^{1,2,5-11,13-15}$ Some of the largest studies were, however, performed partly or fully without the benefit of essential modern surgical instrumentation like endophotocoagulation, dual-function vitreous cutters, and wide-angle viewing systems. ${ }^{2,7,8}$ Vitrectomy was generally performed after long waiting periods, good results were mostly obtained in the presence of total PVD, intraoperative lensectomy was frequent, and poor outcomes were reported with preoperative or intraoperative detachments. The overall surgical results were still called 'satisfactory' because a majority of cases were operated for $\mathrm{VH}$ and had complete PVD. ${ }^{1,2,7,8,13,14}$ However, PVD is not necessarily common in Eales' disease, and PPV not as straightforward as claimed by many authors. ${ }^{1,2,8}$ Badrinath et $a l^{12}$ found an incomplete PVD in all the 18 eyes operated for Eales' disease. They warned about frequent vitreoschisis in Eales' disease, resulting in 


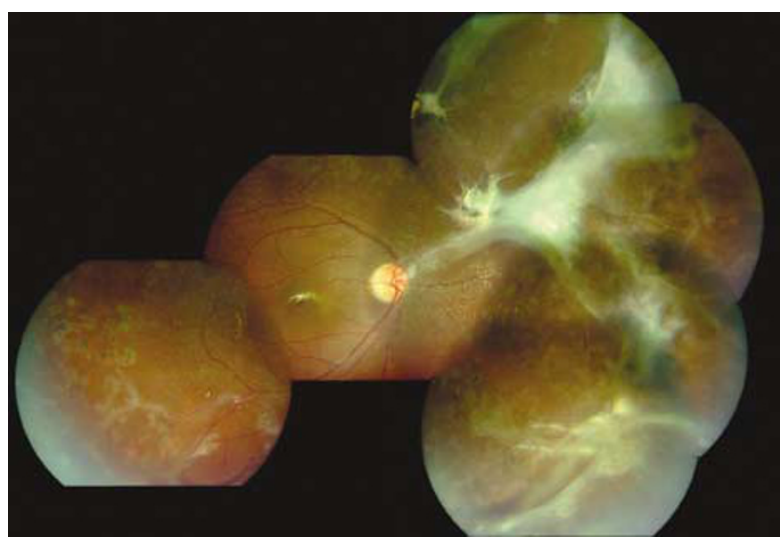

Figure 1 A composite fundus view of a case of Eales' disease 6 months after vitrectomy, showing a healthy macula in spite of a large nasal residual tractional band with multiple attachments. Sheathed vessels are seen in the vicinity of the band as well as inferotemporally. Peripheral scatter photocoagulation marks are visible all around. Indentation of the encircling band is seen inferonasally.

residual tangential traction if not delaminated. ${ }^{15}$ This discrepancy in surgical experience is evident in more recent studies as well. Kumar $e t a l^{6}$ reported excellent visual outcome in Eales' disease ( $\geqslant 6 / 18$ in $92 \%$; PVD: 70-100\%), ${ }^{6}$ whereas El-Asrar and Al-Kharashi ${ }^{5}$ reported acuity $\geqslant 6 / 12$ in a modest $26 \%$ (incidence of PVD not reported; preoperative RD in 27\%). Majji et al ${ }^{9}$ had final acuity $\geqslant 20 / 50$ in $30 \%$ cases, $28 \%$ ended up legally blind (membrane peeling was performed in all, suggesting incomplete PVD). A recent long-term study reported dismal results with vitrectomy in Eales' disease (functional improvement in only 26.5\%). ${ }^{10}$ Another recent study reported final acuity of $\geqslant 20 / 50$ in $51 \%$; but $42 \%$ presented with the same vision preoperatively. ${ }^{11}$ In spite of this wide range of results, we are unaware of any previous study comparing surgical results obtained with/without PVD or RD. We obtained a final $\mathrm{BCVA} \geqslant 6 / 12$ in $43(60.6 \%)$ eyes, notwithstanding an incomplete PVD in $60.6 \%$ eyes; only three $(4 \%)$ of our cases had BCVA $\geqslant 6 / 12$ preoperatively. The presence of preoperative RD in only a third of eyes probably improved the prognosis. But absence of RD did not necessarily mean a simple surgery: adjunctive procedures $\left(\mathrm{SB} / \mathrm{C}_{3} \mathrm{~F}_{8} /\right.$ silicone oil) were required in $31(43.7 \%)$ cases for epiretinal membranes (Figure 1). Indeed, the mean final visual acuity was not significantly different in the groups with VH and RD. Preoperative PRP did not affect the surgical outcome, contrary to the general opinion. . $^{1,26-8}$ This anomaly may be attributed to the small number of PRP-treated eyes $(n=21)$. It is also probable that PRP was performed a little too late, when fibrovascular tractional membranes were already established.
We had a relatively high incidence of postoperative cataract formation (24\%), which is, however, comparable to that reported in other long-term studies., ${ }^{711}$ This was due probably to additional surgical procedures $\left(\mathrm{SB} / \mathrm{C}_{3} \mathrm{~F}_{8} /\right.$ silicone oil) having been required in $31(43.7 \%)$ cases. Our surgical failure rate (15.5\%) was comparable to other studies, ${ }^{7-11}$ and was mostly related to the difficulty of peeling tractional membranes in the peripheral retina. Peripheral tears or retinotomies were observed in all the persistent/inoperable detachments. In the final analysis, 14 (19.7\%) eyes ended up with final BCVA $\leqslant 3 / 60$. Shanmugam $e t a l^{7}$ had $29.6 \%$ eyes with a similar fate. It is noteworthy that out of 16 eyes, which underwent belt buckling as a primary or secondary procedure, only two were anatomical failures. Although the number of procedures is too small to draw significant conclusions, encirclage appears to help in neutralising peripheral traction from fibrovascular membranes.

Our study had the limitations of a retrospective, uncontrolled case series. The cases were also nonconsecutive, as a minimum follow-up of six months was an entry criterion. As the follow-up varied considerably (6-72 months), it may be difficult to compare follow-up outcomes in individual patients. However, the mean BCVA score had already improved significantly from the baseline by 6 months in $90 \%$ of our cases (5.92 (4.56, 7.27); $P<0.0001)$ and there was no significant difference in the scores at 6 months and at the last follow-up ( $P=0.5388)$. Similarly, others have also reported an early stabilisation of visual outcomes, which changed little over a long-term follow-up. ${ }^{7}$ Further, the surgical techniques were more or less consistent over this 8-year period, and involved the use of state-of-the-art technology (wide-angle viewing systems, endophotocoagulation, modern vitreous cutters, etc); a major advantage over other large, long-term studies. Our surgical experience with Eales' disease can be summed up as follows: we did not observe a preoperative PVD as commonly as generally presumed; absence of preoperative RD does not necessarily mean a straightforward vitrectomy, as peripheral tractional sequelae frequently coexist with $\mathrm{VH}$; and encircling belt buckle may help to improve the anatomical outcome in presence of peripheral membranes. Finally, we demonstrate that good surgical outcomes are possible in Eales' disease, even in the presence of tractional sequelae and absence of PVD.

\section{References}

1 Biswas J, Sharma T, Gopal L, Madhavan HN, Sulochana KN, Ramakrishnan S. Eales disease - an update. Surv Ophthalmol 2002; 47: 197-214. 
2 Das T, Biswas J, Kumar A, Nagpal PN, Namperumalsamy P, Patnaik B et al. Eales' disease. Indian J Ophthalmol 1994; 42: 3-18.

3 Nussenblatt RB, Whitcup SM, Palestine AG. Retinal vasculitis. In: Nussenblatt RB, Whitcup SM, Palestine AG (eds). Uveitis Fundamental and Clinical Practice, 2nd ed Mosby: St Louis, 1996, pp 354-363.

4 Patnaik B, Nagpal PN, Namperumalsamy P, Kalsi R. Eales' disease: clinical features, pathophysiology, etiopathogenesis. Ophthalmol Clin North Am 1998; 11: 601-617.

5 El-Asrar AM, Al-Kharashi SA. Full panretinal photocoagulation and early vitrectomy improve prognosis of retinal vasculitis associated with tuberculoprotein hypersensitivity (Eales' disease). Br J Opthalmol 2002; 86: 1248-1251.

6 Kumar A, Tiwari HK, Singh RP, Verma L, Prasad N. Comparative evaluation of early vs deferred vitrectomy in Eales' Disease. Acta Ophthalmol Scand 2000; 78: 77-78.

7 Shanmugam MP, Badrinath SS, Gopal L, Mahesh P, Sharma $\mathrm{T}$. Long term visual results of vitrectomy for Eales disease complications. Int Ophthalmol 1998; 22: 61-64.

8 Atmaca LS, Nagpal PN. Eales' disease: medical, laser, and surgical treatments. Ophthalmol Clin North Am 1998; 11: 619-626.
9 Majii AB, Vemuganti GK, Shah VA, Singh S, Das T, Jalali S. A comparative study of epiretinal membranes associated with Eales' disease: a clinicopathologic evaluation. Eye 2006; 20: $46-54$

10 Smiddy WE, Isernhagen RD, Michels RG, Glaser BM, De Bustros SN. Vitrectomy for nondiabetic vitreous hemorrhage: retinal and choroidal vascular disorders. Retina 1988; 8: 88-95.

11 Treister G, Machemer R. Results of vitrectomy for rare proliferative and hemorrhagic disease. Am J Opthalmol 1977; 84: $394-412$

12 Badrinath SS, Gopal L, Sharma T, Parikh S, Shanmugam MP, Bhende $\mathrm{P}$ et al. Vitreoschisis in Eales' disease: pathogenic role and significance in surgery. Retina 1999; 19: 51-54.

13 Mason III JO, Colagross CT, Haleman T, Jeffrey J, White MF, Feist RM et al. Visual outcome and risk factors for light perception and no light perception vision after vitrectomy for diabetic retinopathy. Am J Ophthalmol 2005; 140: 231-235.

14 Atmaca LS, Batioglu F, Sonmez PA. A long-term follow-up of Eales' disease. Ocul Immunol Inflam 2002; 10: 213-221.

15 Dehghan MH, Ahmadieh H, Soheilian M, Azarmina M, Mashayekhi A, Naghibozakerin J. Therapeutic effects of laser photocoagulation and/or vitrectomy in Eales' disease. Eur J Ophthalmol 2005; 15: 379-383. 